\title{
QCM Gas Sensor with Organic Nanowire Film as Molecular Recognition Membrane
}

\author{
Yoshiaki Dehari ${ }^{1}$, Yoshinori Takei ${ }^{1,2, *}$, Shin Koyama ${ }^{2}$, \\ Hidehito Nanto ${ }^{1,2}$ and Shuhei Seki ${ }^{3}$ \\ 'Department of Robotics, Kanazawa Institute of Technology, \\ 7-1 Ohgigaoka, Nonoichi, Ishikawa, 921-8501, Japan \\ ${ }^{2}$ Research Laboratory for Integrated Technological Systems, Kanazawa Institute of Technology, \\ 3-1 Yatsukaho, Hakusan, Ishikawa, 924-0838, Japan \\ ${ }^{3}$ Division of Applied Chemistry, Graduate School of Engineering, Osaka University, \\ 2-1 Yamadaoka, Suita, Osaka, 565-0871, Japan
}

(Received January 4, 2010; accepted April 12, 2010)

Key words: quartz crystal microbalance, recognition membrane, nanowires, plasma CVD, gas sensor

In this paper, we demonstrate a novel quartz crystal microbalance (QCM) gas sensor with nanowires of a molecular recognition membrane (MRM), which is fabricated by the nanosize particle-beam irradiation technique. It was found that the QCM sensor with poly-acrylic acid MRM exhibited an excellent selectivity for ammonia gas. It is confirmed that the sensitivity of the QCM sensor with nanowires of poly-acrylic acid MRM is higher than that of the sensor with a common poly-acrylic acid MRM. Furthermore, we investigate the influence of the functional group of MRM on the gas sensing characteristics of the QCM gas sensor.

\section{Introduction}

A quartz crystal microbalance (QCM) can be a sensitive mass measuring device in nanogram levels because of the fact that the resonant frequency changes upon the deposition of a given mass on the electrode of the QCM. The QCM coated with synthetic polymer films has been investigated for use as a gravimetric gas sensor capable of detecting various gases. ${ }^{(1)}$ These types of gas sensor can be characterized using coated polymer films, which we call the film molecular recognition membranes (MRMs).(2)

In this paper, we show a QCM gas sensor with MRMs fabricated by the nanosize particle-beam irradiation technique.(3) It was found that the QCM sensor with polyacrylic acid MRMs exhibits excellent selectivity for ammonia gas.(2) It is confirmed that the sensitivity of the QCM sensor with nanowires of poly-acrylic acid MRM is higher than that of the sensor with a common poly-acrylic acid MRM.

*Corresponding author: e-mail: takei@neptune.kanazawa-it.ac.jp 
Furthermore, we study the influence of the functional group of MRM on the gas sensing characteristics of the QCM gas sensor. The relationship between the functional group contained in the MRM and gas sensitivity was considered in the previous work..$^{(2)}$ Here, the fluoro functional group is investigated, and it shows that the MRMs with the functional group are sensitive to aldehyde gases.

\section{Experimental}

A commercially available AT-cut $9 \mathrm{MHz}$ QCM with $\mathrm{Au}$ electrodes was prepared, which is utilized as a gas sensor coated with the MRM on the surface. The MRMs can be fabricated by two procedures.

The nanowired type of MRM was fabricated by the nanosize particle-beam irradiation technique. First, as the base, a polymer material such as poly-acrylic acid was coated on the electrode of the QCM by the dipping method. It is based on the result that a sensor with an acrylic acid membrane exhibits sensitivity to ammonia gas. ${ }^{(2)}$ The polymer was irradiated with a $450 \mathrm{MeV} \mathrm{Xe}$ ion beam. High-energy particles penetrating into the polymer effectively promote cross-linking reaction along trajectories. Figure 1 shows a schematic view of an ion track in polymer materials. After the irradiation, noncrosslinking polymers are dissolved by the permeation of a solvent such as dioxane. Then, nanowires that have a cylindrical structure of cross-linked polymers are formed on the QCM.

We also use plasma chemical vapor deposition (CVD) to coat the substrate of the QCM with monomers, which have a fluoro functional group. The MRMs were deposited on the electrode of the QCM by the plasma polymerization of monomers such as 2.2.2-trifluoroethanol, 4-chlorobenzotrifluoride, 2.2.2-trifluoroethyl acrylate,

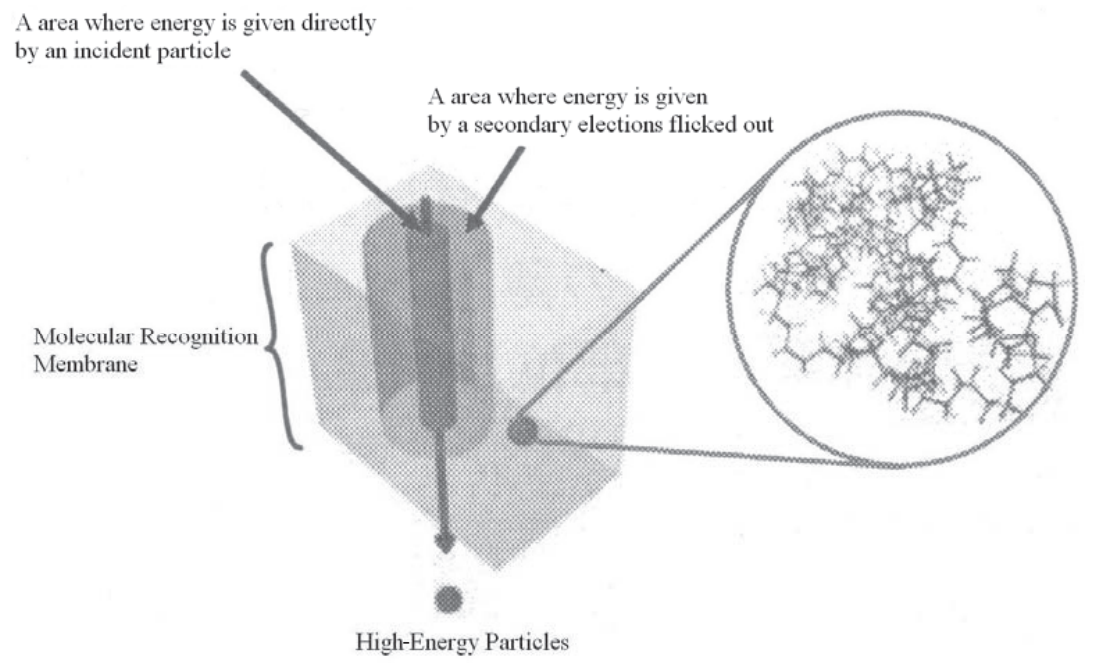

Fig. 1. Schematic view of an ion track in polymeric materials. 
benzotrifluoride, and 2.2.2-trifluoroethyl methacrylate at a pressure of $100 \mathrm{~Pa}$ with an $\mathrm{rf}$ power of $100 \mathrm{~W}$. The monomer temperature and deposition time are $40^{\circ} \mathrm{C}$ and $150 \mathrm{~min}$, respectively.

Figure 2 shows the schematic diagram of the QCM sensing system. The QCM sensors are connected to a PC via a frequency counter (THQ-10DA, Hokkaido Toukakeiki K.K.), and the frequency change caused by the adsorption of gases injected using a microsyringe into the chamber is measured. The temperature and humidity in the chamber are controlled at $20 \pm 1{ }^{\circ} \mathrm{C}$ and $20 \pm 5 \%$, respectively. A constant amount of gases is injected into the sensing chamber. Aldehydes such as acetaldehyde, glutaraldehyde, formaldehyde, propionaldehyde, acrolein, benzaldehyde, and cinnamaldehyde are prepared at 10,000 ppm for the MRMs containing the fluoro functional group, and ammonia is prepared at $1,250 \mathrm{ppm}$ for the nanowired MRM.

\section{Results and Discussion}

Figures 3 to 7 show typical transient responses to aldehydes obtained with the QCM gas sensors coated with the MRMs containing the fluoro functional group. The sensing chamber was opened 3 min after injecting the gases.

Each response shows that the gas sensor with the fluoro functional MRM exhibits excellent sensitivity to aldehydes, particularly, acetaldehyde, propionaldehyde, and acrolein. It is seen that the fluoro functional MRMs can be utilized as the sensing membrane for aldehyde gases.

The developed sensors show lower sensitivity to some aldehydes, which are mainly innoxious. The sensor with a 2.2.2-Trifluoroethanol membrane responds to acetaldehyde with a small time constant as compared with the response to propionaldehyde, as shown in Fig. 3, and a similar characteristic can be seen in the other responses.

Figure 8 shows typical transient responses of the sensor with the nanowired MRM and the sensor with the dip-coated MRM to ammonia gases. It can be seen that both

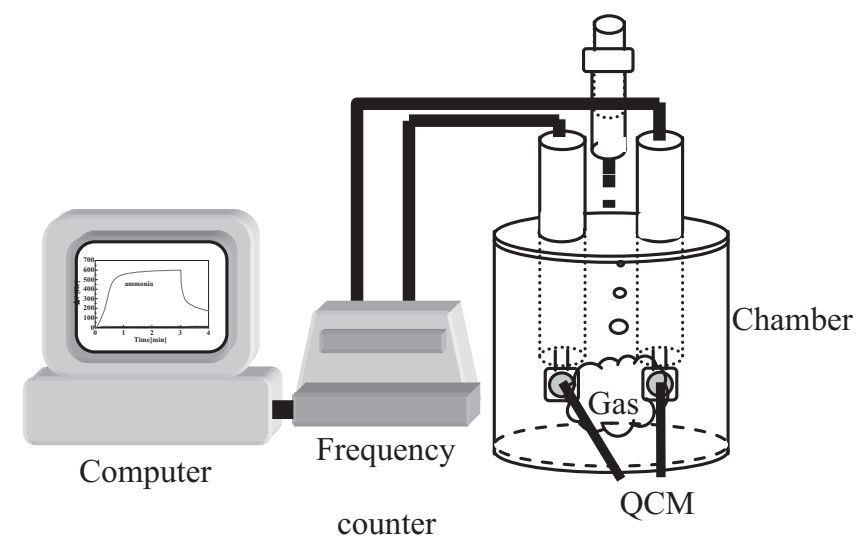

Fig. 2. Schematic diagram of QCM sensing system. 


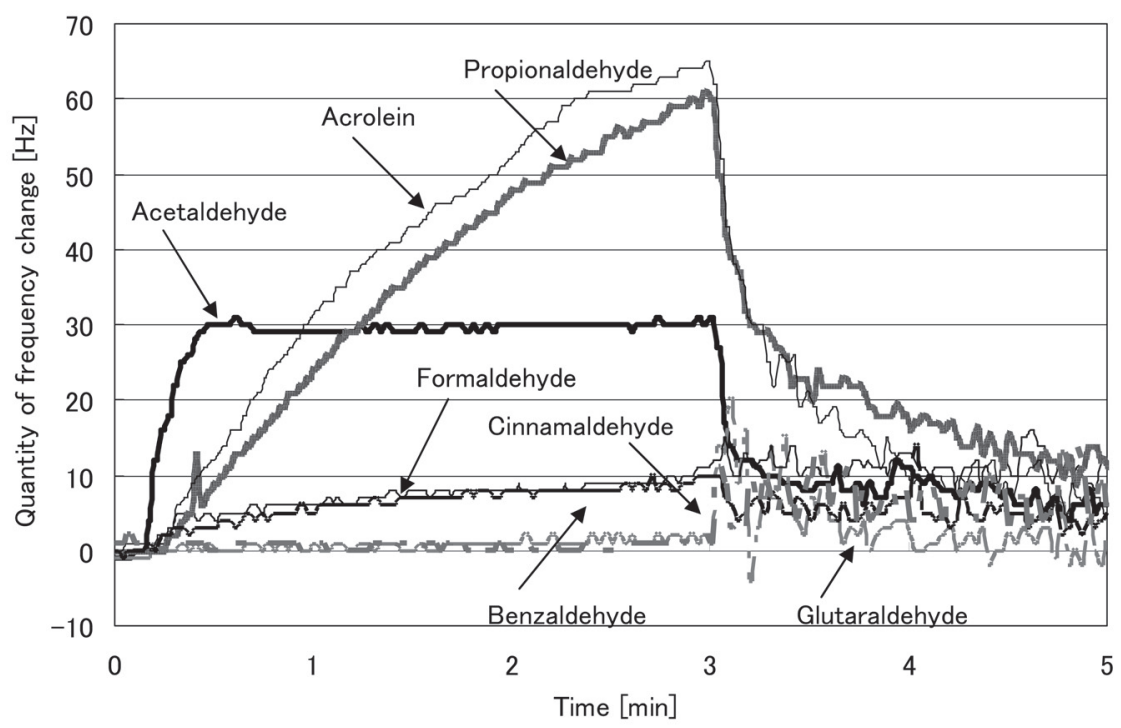

Fig.3. Typical transient responses of the 2.2.2-trifluoroethanol membrane to aldehyde gases.

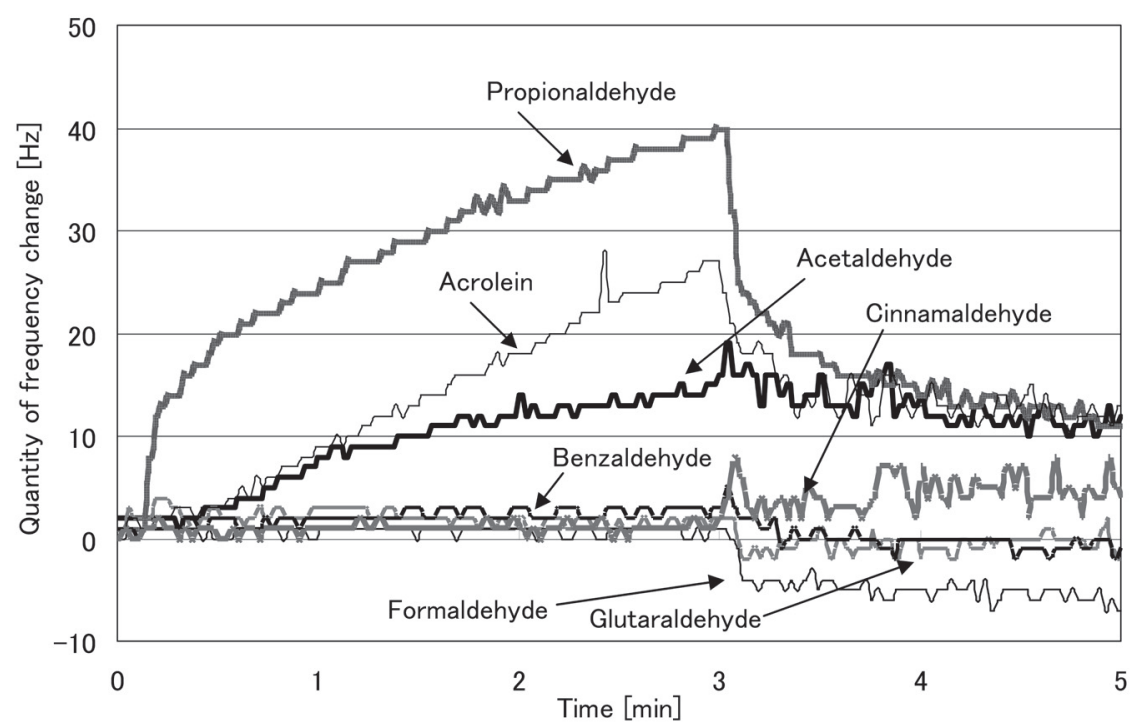

Fig. 4. Typical transient responses of the 4-chlorobenzotrifluoride membrane to aldehyde gases. 


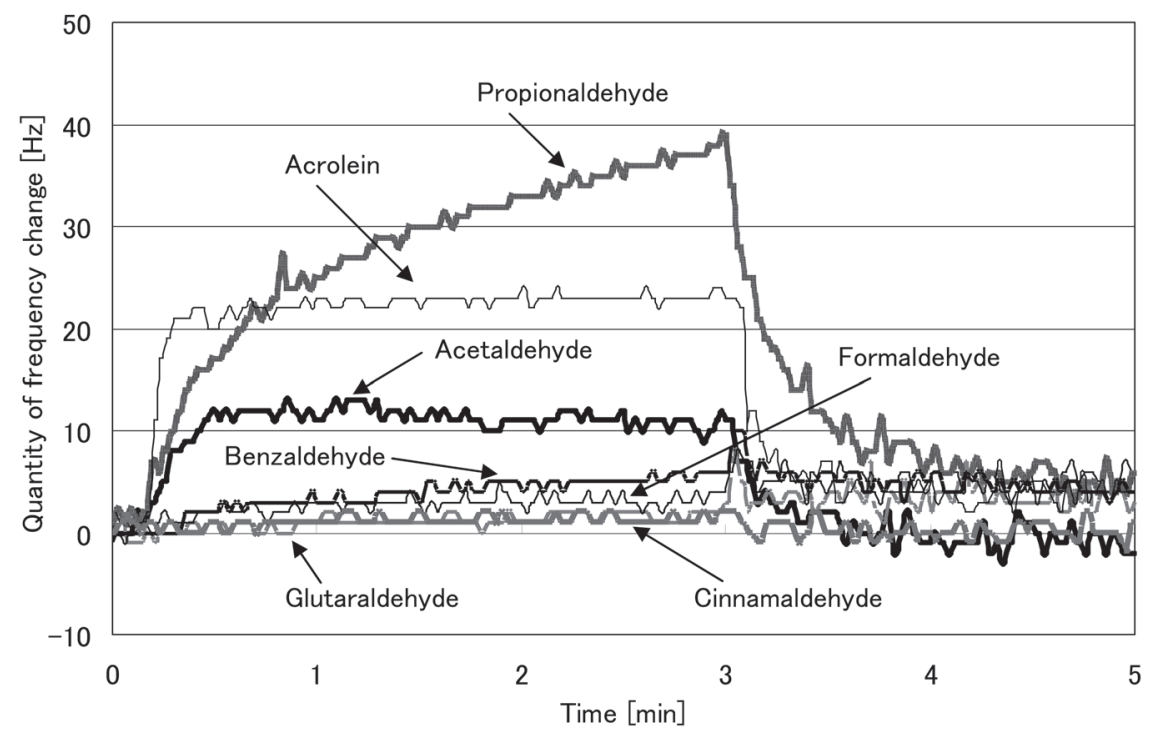

Fig. 5. Typical transient responses of the 2.2.2-trifluoroethyl acrylate membrane to aldehyde gases.

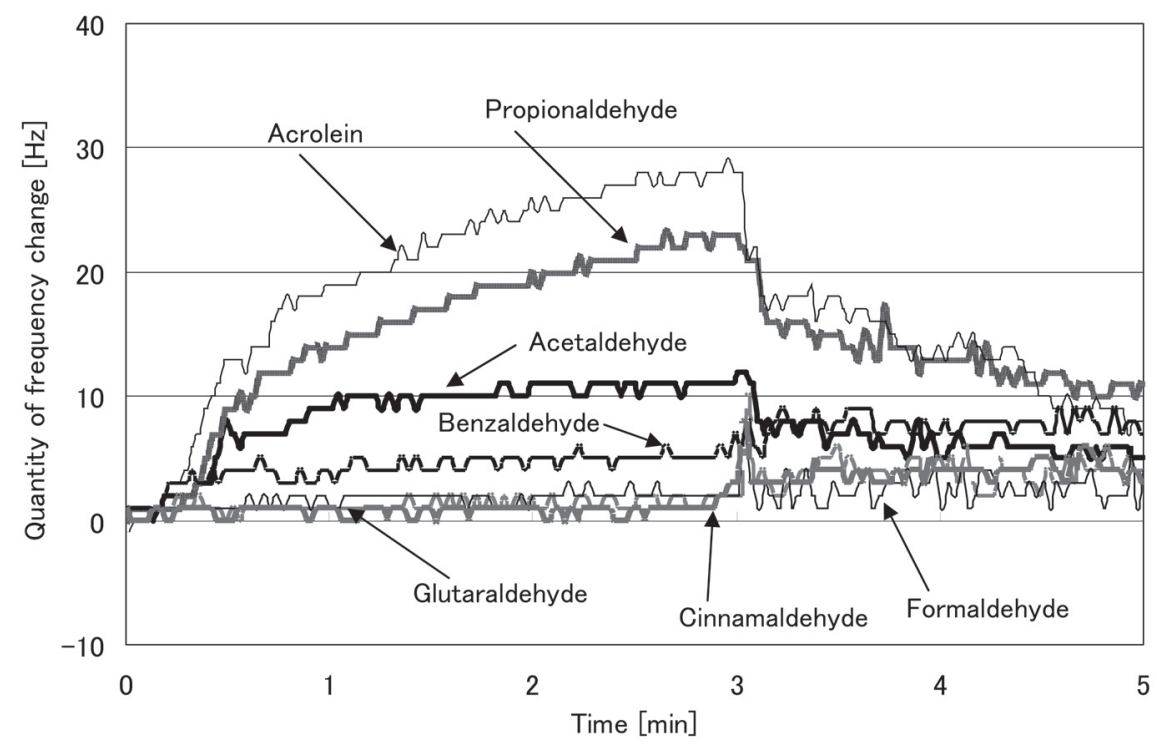

Fig. 6. Typical transient responses of the benzotrifluoride membrane to aldehyde gases. 


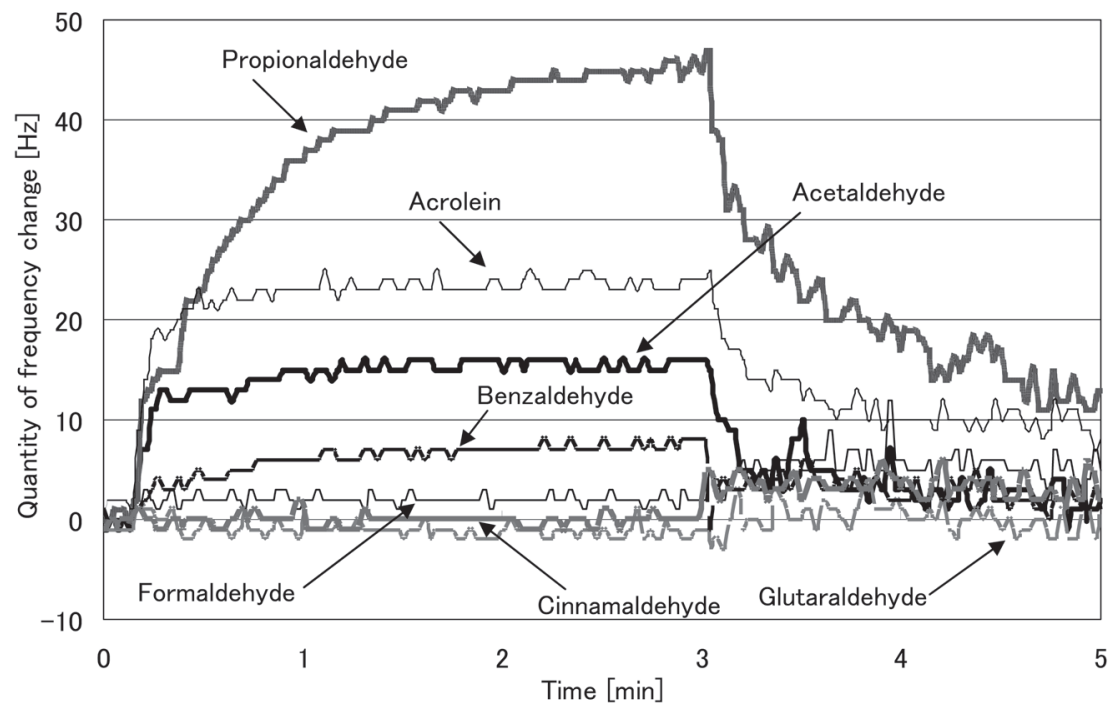

Fig. 7. Typical transient responses of the 2.2.2-trifluoroethyl methacrylate membrane to aldehyde gases.

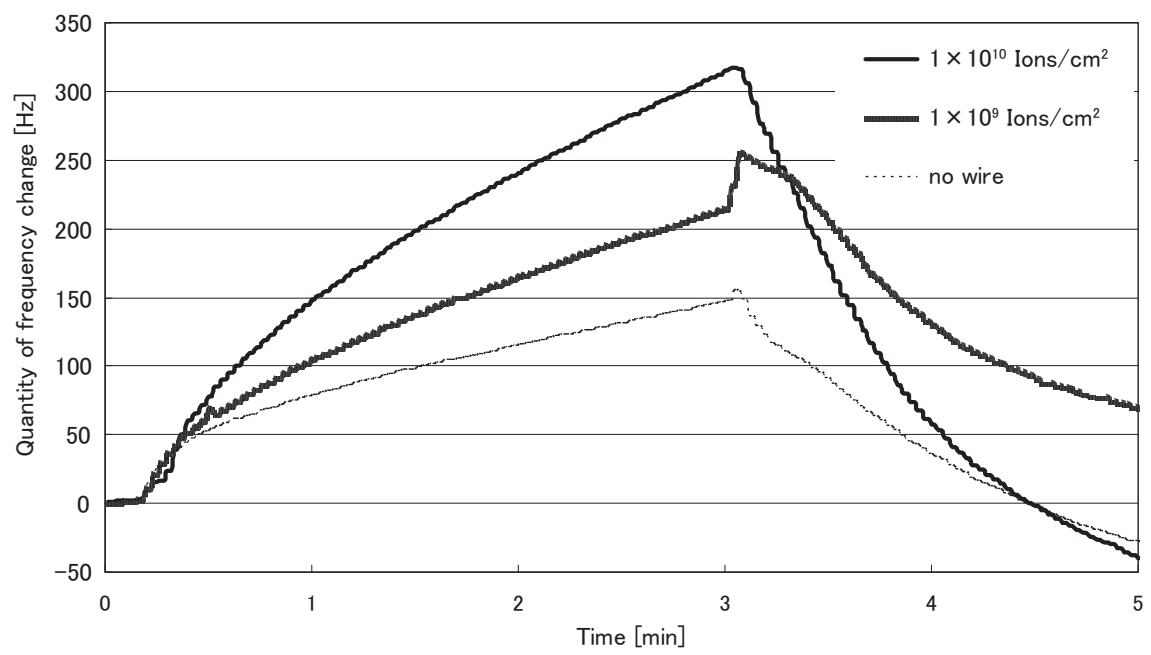

Fig. 8. Typical transient responses of the nanowire membrane to ammonia gases. 
sensors with the nanowired and dip-coated MRMs exhibit good sensitivity to ammonia gas and that the sensitivity of the dip-coated MRM sensor can be improved by nanowiring the MRM. It is also found that the sensitivity of the nanowired MRM sensor can be improved by increasing the number of nanowires.

\section{Conclusions}

In this paper, we demonstrated a novel QCM gas sensor with nanowires of MRM, which is fabricated by the nanosize particle-beam irradiation technique. We showed that the QCM sensor with nanowires of poly-acrylic acid MRM exhibits excellent sensitivity to ammonia gas, and that the sensitivity of the nanowire sensor can be higher than that of the sensor with a common poly-acrylic acid MRM. Furthermore, we investigated the influence of the functional group of MRM on the gas sensing characteristics of the QCM gas sensor. We have shown that fluoro functional MRMs can be utilized as sensing membranes for aldehyde gases.

\section{Acknowledgement}

This work was partly supported by the Open-Research Foundation from the Ministry of Education, Culture, Sports, Science and Technology of Japan.

\section{References}

1 T. C. Pearce et al.: Handbook of Machine Olfaction (Wiley-VCH, Weinheim, 2003) pp. 89-91.

2 H. Nanto, Y. Takei, T. Imamura and D. Ishigure: Proc. NSTI Nanotech 3 (2006) 475.

3 S. Seki: Ionizing radiation 34 (2008) 31. 